\title{
Laboreal
}

Volume $14 \mathrm{~N}^{\circ} 2$ | 2018

Digitalização e evolução do trabalho real

\section{Revisão crítica de publicações científicas sobre acidentes de trabalho fatais na indústria do petróleo e gás}

Revisión crítica de publicaciones científicas sobre accidentes de trabajos fatales en la industria del petróleo y gas

Revue critique de publications scientifiques sur les accidents du travail mortels dans l'industrie du pétrole et du gaz

A critical literature review of fatalities in gas and oil industry

Hilka Guida, Élida Azevedo Hennington e Marcelo Figueiredo

\section{(C) OpenEdition}

\section{Edição electrónica}

URL: http://journals.openedition.org/laboreal/665

DOI: $10.4000 /$ laboreal. 665

ISSN: 1646-5237

\section{Editora}

Universidade do Porto

\section{Refêrencia eletrónica}

Hilka Guida, Élida Azevedo Hennington e Marcelo Figueiredo, « Revisão crítica de publicações científicas sobre acidentes de trabalho fatais na indústria do petróleo e gás », Laboreal [Online], Volume $14 \mathrm{~N}^{\circ} 2$ | 2018, posto online no dia 01 dezembro 2018, consultado o 06 outubro 2019. URL http://journals.openedition.org/laboreal/665; DOI : 10.4000/laboreal.665

Este documento foi criado de forma automática no dia 6 outubro 2019.

Laboreal está licenciado com uma Licença Creative Commons - Atribuição-NãoComercial 4.0 Internacional. 


\section{Revisão crítica de publicações científicas sobre acidentes de trabalho fatais na indústria do petróleo e gás}

Revisión crítica de publicaciones científicas sobre accidentes de trabajos fatales en la industria del petróleo y gas

Revue critique de publications scientifiques sur les accidents du travail mortels dans l'industrie du pétrole et du gaz

A critical literature review of fatalities in gas and oil industry

Hilka Guida, Élida Azevedo Hennington e Marcelo Figueiredo

\section{NOTA DO EDITOR}

http://dx.doi.org/10.15667/laborealxiv218hg

Manuscrito recebido em: maio/2018

Aceite após peritagem: outubro/2018

\section{Introdução}

1 A Organização Internacional do Trabalho (1981) declarou como direito fundamental dos trabalhadores a segurança e saúde no trabalho. Essa agência ressalta a importância da proteção dos trabalhadores contra a ocorrência de acidentes de trabalho e aponta a necessidade de sua redução como um elemento imprescindível para a justiça social. Os ambientes de trabalho devem necessariamente ser locais com condições dignas, saudáveis e seguras. Sendo assim, é imperativo o enfrentamento dos acidentes de 
trabalho, pois matam e lesionam mais do que grandes guerras, embora não costumem ser destacados pela mídia.

A palavra 'acidente', de acordo com o Dicionário Aurélio (Ferreira, 2002), é substantivo masculino que significa 'acontecimento casual, fortuito, imprevisto'. Durante muitos anos e, ainda hoje, em visões mais conservadoras, o acidente de trabalho é tido como algo não previsto, obra do azar, sem intencionalidade (Santana et al., 2007).

De acordo com dados da OIT (2013), cerca de 313 milhões de trabalhadores sofrem acidentes de trabalho por ano e 6.400 pessoas morrem em decorrência de acidentes ou doenças profissionais por dia no mundo inteiro, resultando em cerca de 2,3 milhões de mortes por ano. Estas ocorrências geram um custo médio de 2,8 trilhões de dólares anuais. O Brasil contribui significativamente e negativamente para estas estimativas, tendo em vista que ocupa o $4^{\mathrm{o}}$ lugar no ranking de ocorrências, atrás apenas de China, Índia e Indonésia (Organização Internacional do Trabalho, 2013).

Segundo o Anuário Estatístico da Previdência Social (Ministério da Fazenda, 2015) houve 612.632 acidentes de trabalho em 2015, representando uma queda de $14 \% \mathrm{em}$ relação a 2014 e 2.502 mortes. Cabe salientar que tal redução se dá em uma conjuntura de profunda recessão, com sensível elevação do desemprego, e pode expressar também um aumento da subnotificação e não a melhoria das condições de segurança e saúde no trabalho. Ressalta-se que nesta estatística não estão contabilizados os casos referentes a trabalhadores excluídos do seguro acidentário tais como: autônomos, empresários, servidores públicos e também os trabalhadores sem vínculo empregatício formal de trabalho e que hoje representam cerca de $50 \%$ da população economicamente ativa brasileira. Verifica-se que $76,3 \%$ são os chamados acidentes típicos, $21 \%$ os acidentes de trajeto e 2,7\% as doenças ocupacionais. Os trabalhadores do sexo masculino representam $70,3 \%$ dos acidentados e as mulheres $29,7 \%$ dos registros de acidentes de trabalho.

5 Este artigo pretende analisar criticamente o perfil de acidentes de trabalho fatais na indústria de petróleo e gás a partir de publicações científicas nacionais e internacionais sobre o tema. Buscou-se identificar as revistas publicadas, as instituições e autores e realizar uma caracterização sobre os acidentes ocorridos, o tipo de ocorrência, o local de ocorrência, o tipo de planta e o número de fatalidades por evento.

\section{Método}

6 Foi realizada revisão bibliográfica do tipo narrativa, em outubro de 2017, na base Scopus, sobre o tema 'acidentes fatais na indústria de petróleo e gás' para identificar, selecionar e, por fim, incluir e analisar estudos nacionais e internacionais relacionados ao tema, seguindo os preceitos dos estudos exploratórios.

\subsection{Estratégia da pesquisa}

7 A identificação dos artigos sobre acidentes de trabalho fatais ocorridos na indústria de petróleo e gás no cenário mundial foi realizada por meio da utilização da base de dados eletrônica Scopus. Esta foi a base que apresentou maior número de estudos dentre as bases utilizadas em pesquisa bibliográfica realizada previamente sobre o tema. 
Os critérios de inclusão adotados e utilizados para a seleção dos artigos foram: estudos em português, espanhol, francês ou inglês sobre acidente de trabalho fatal envolvendo a indústria de petróleo e gás, publicados em qualquer período em revista indexada. Foram excluídos estudos sobre ocorrência de morte na indústria de petróleo e gás por doença, seja ela ocupacional ou doença comum, ocorrência de acidentes não relacionados ao trabalho e ocorrência de acidentes que atingiram exclusivamente a comunidade do entorno da instalação de petróleo e gás.

Os descritores/termos utilizados foram obtidos a partir do vocabulário DeCs e MeSH fornecidos pelas bases Bireme e Pubmed, respectivamente. Foi utilizada a seguinte estratégia de busca e respectivos descritores e operadores boleanos: KEY (petroleum OR 'oil and gas fields' OR 'petroleum industry' OR 'oil and gas industry' OR 'extractive industry' OR 'natural gas' OR petrochemical OR 'Offshore oil wells' OR 'Petroleum deposits' OR 'Petroleum engineering' OR 'oil refinery') AND TITLE-ABS ('accidental falls' OR 'work accident' OR 'risk factors' OR 'occupational injuries' OR 'accidents' OR 'wounds and injuries' OR 'accidents, occupational' OR 'Catastrophic accidents' OR 'Catastrophic consequences' OR 'Accident prevention') and title-abs-key ('mortality' OR 'death' OR 'fatality' OR 'Fatal accident' OR 'Occupational fatality') AND NOT ('disease*'OR comorbidity). Todo o processo de busca, seleção e extração dos dados dos artigos foi feito utilizando como ferramenta o software Zotero.

Após a busca inicial, foram identificados e selecionados 198 artigos a partir dos títulos e resumos. Posteriormente, obteve-se o texto na íntegra de todos os artigos, sendo selecionados e analisados 42 artigos ao final.

\subsection{Síntese dos dados}

11 A partir dos artigos selecionados, realizou-se a tabulação e análise dos dados, incluindo informações sobre as características gerais e específicas dos estudos. Os seguintes itens foram incluídos e agrupados: ano de publicação, tipo de publicação, autoria, área de conhecimento, nome da revista, ano de publicação, idioma, localização/região da realização do estudo, população, objetivo principal do estudo, método, número de mortes, descrição do acidente e os principais resultados.

\subsection{Etapas da Busca}

A Figura 1 mostra o processo de seleção e o número de artigos encontrados em cada etapa da busca: 
Figura 1 - Etapas do levantamento bibliográfico sobre acidentes fatais na indústria de petróleo e gás, base Scopus, outubro de 2017

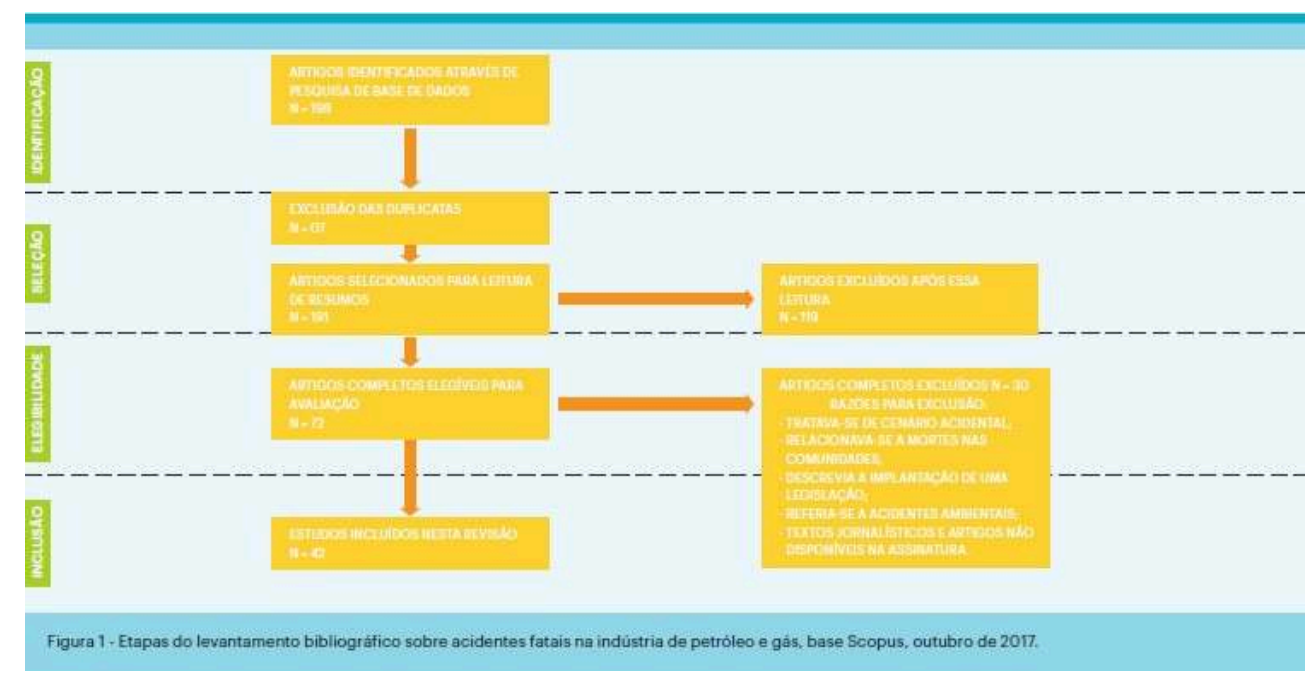

\section{Resultados e discussão}

Foram selecionados 42 artigos que abordam os acidentes fatais na indústria de petróleo e gás no cenário nacional e internacional (Ambituuni, Amezaga \& Werner, 2015a, 2015b; Attwood, Khan \& Veitch, 2006; Bariha, 2016; Bhalla; 2010; Burgherr, Eckle \& Hirschberg, 2012; Cabral, Pinheiro, Marrozzi, \& Marchi, 2010; Catten \& Johnson, 2014; Childress, 2014; Covil \& Smith, 2002; Curlee, Broulliard, Marshall, Knode, \& Smith, 2005; Denkl, Anderson \& Marley, 2010; Ebrahimian \& Stefanescu, 2011; Eckle \& Burgherr, 2013; Egbeocha, Reginald-Ugwuadu, Oluchi, Ebisike, \& Obanya, 2015; Espinosa et al., 2010; Ezenwa, 2001; Fullerton, Olson, Crandall, Sklar, \& Zumwalt, 1995; Goyal, 2010; Hill, Somervell \& Conway, 2009; Hought, 2010; Igbuku, 2007; Ismail, Ramli \& Somarin, 2012; Jutten, Ndubisi, Regnault, \& Abifarin, 2002; Khan \& Abbasi, 1999; Leistad \& Bradley, 2009; Lightfoot \& Susler, 2006; Lindøe, Engen \& Olsen, 2011; Martinez, 2013, Odunmbaku \& Obarewon, 2007; Piccinini \& Demichela, 2012; Powell, 1996; Riezel, 2002; Schmidt, 2013; Sharma, Gurjar, Singhal, \& Agrawal, 2015; Sherman, 2009; Skelton, 2014; Smith, 2002; Stuart \& Foo, 2010; Threadgold, Jeffrey, Sixma, \& Garcia, 2010; Valner \& Leighton, 2009; Wilson \& Gallagher, 2005). Tais artigos foram analisados em sua íntegra e os resultados são apresentados a seguir.

Os acidentes reportados ocorreram no período de 1965 a 2013. Em relação ao número de artigos publicados por ano identificou-se uma tendência de aumento substancial nas últimas duas décadas. Os anos que apresentaram o maior número de publicações foram 2002, 2009, 2010 e 2015. Verifica-se que o maior número de publicações se refere à ocorrência de três grandes acidentes ampliados nos EUA, oque pode ter impulsionado o aumento de publicações nestes anos. No ano de 2001 também houve4acidentes de alto impacto na indústria de petróleo e gás, sendo um na França com 30 fatalidades e, no Brasil, em uma plataforma de petróleo com 11 mortes. 


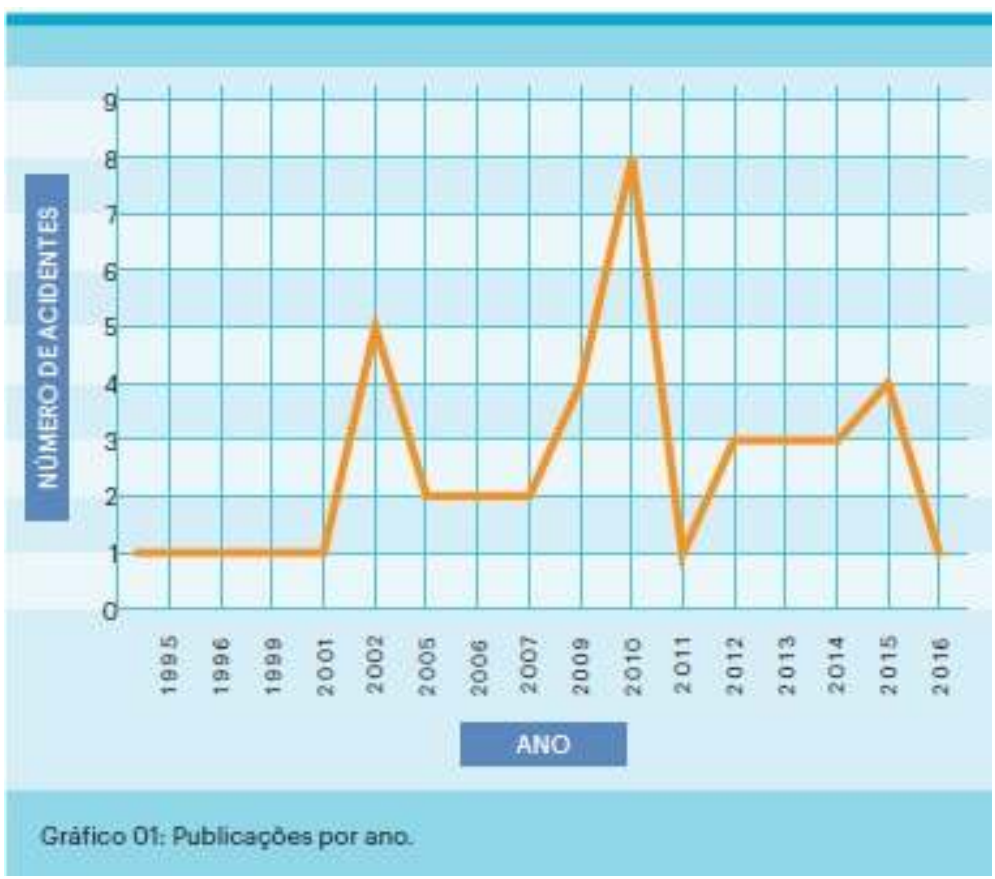

Cerca de 91\% das publicações são das últimas duas décadas (de 2000 a 2016), o que demonstra que a revisão apresentada traz o debate recente sobre a ocorrência de acidentes de trabalho fatais na indústria de petróleo e gás. Além disso, denota que têm aumentado o interesse dos pesquisadores no estudo das fatalidades neste setor e em suas consequências.

Desses 42 artigos científicos, 54,8\% utilizavam metodologia qualitativa e 45,2\% utilizavam metodologia quantitativa o que evidencia que não há predominância de determinado desenho metodológico na amostra analisada. Dos 23 artigos classificados como de abordagem qualitativa identificou-se que 15 buscavam realizar análises a partir do relato de experiência de um acidente ocorrido ou do desenvolvimento de programa implementado por empresas do ramo de petróleo e gás para redução das ocorrências fatais. Destes, quatro eram estudos de revisão de literatura e um estudo de caso.

No que se refere à área de conhecimento das publicações identificou-se que 20 artigos (47,6\%) dos estudos foram publicados na área de Petróleo e Gás; na Engenharia foram19 artigos (42.8\%), 2 na área de Saúde $(4,8 \%)$ e 1 na Ciência da Navegação $(2,4 \%)$. Nas áreas de Petróleo e Gás e Engenharia houve maior destaque para os campos de conhecimentos de Segurança do Trabalho e Segurança de Processo. Os da área da Saúde foram estudos epidemiológicos dos EUA e Nigéria, publicados nos anos de 1995 e 2001, evidenciando assim a escassez de pesquisas e debate do tema pelo campo Saúde.

\subsection{Revistas publicadas}

18 Identificou-se que cerca de $50 \%$ das publicações estão concentradas em um único editorial, o da revista da Society of Petroleum Engineers (EUA) que, em geral, publica trabalhos técnico-científicos apresentados em conferências promovidas pela própria instituição. Identifica-se esta publicação como a opção preferencial por parte das próprias empresas da área de petróleo e gás, sendo reconhecida como de alta relevância 
e prestígio no meio empresarial. É de acesso restrito e há necessidade de pagamento de assinatura, o que pode constituir em fator limitante na disseminação do conhecimento a respeito do tema. Este editorial possui fator de impacto de 2.21, de acordo com dados da rede ResearchGate. Em segundo lugar, ainda no quesito maior número de publicações sobre o tema estão a Safety Science (EUA), com 3 publicações $(7,1 \%)$ e fator de impacto de 3,11, de acordo com dados da rede ResearchGate e a Process Safety Progress (EUA) com fator de impacto de 0.48 , de acordo com dados da rede ResearchGate. Em terceiro, com 2 publicações (4,8\%), o Journal of Loss Prevention in the Process Industries (Países Baixos) com fator de impacto de 1.62, de acordo com dados da rede ResearchGate.

\subsection{Instituições e autores}

19 Verifica-se que há estudos das mais diversas instituições, como empresas do ramo e universidades e cerca de $24 \%$ (10) dos estudos foram realizados por meio de parceria de investigadores de diferentes instituições. Todos os artigos identificados estavam escritos na língua inglesa e foram publicados, principalmente, em periódicos norteamericanos.

Os maiores produtores de petróleo do mundo são EUA, Arábia Saudita, Rússia, Irã, Iraque, Canadá, Emirados Árabes, China, Kuwait, Brasil, México, Venezuela, Nigéria, Noruega e Catar (Instituto Brasileiro de Petróleo, Gás, e Biocombustível, 2016). Observouse que não há correlação direta entre quantidade de produção científica sobre o tema em foco e os maiores países produtores de petróleo e gás.. Dos maiores produtores de petróleo do mundo na atualidade, quatro figuram na lista de países com publicação sobre o tema, são eles: EUA, Nigéria, Brasil e Noruega.

21 Cerca de $23 \%$ das publicações (10 artigos) são de autores norte-americanos. Além da tradicional hegemonia dos EUA no campo da publicação científica, um fator que possivelmente contribua para o maior interesse na investigação e publicação das fatalidades relacionadas ao trabalho pode ser $\mathrm{o}$ alto custo das indenizações neste país. $\mathrm{O}$ segundo país em número de publicações é a Nigéria (6 artigos), sendo este, em âmbito mundial, o primeiro em número de fatalidades na área de petróleo e gás (Eckle \& Burgherr, 2013). Em terceiro aparece o Reino Unido (4 artigos) e, em seguida, a Índia com 3 artigos. Chama a atenção que o Brasil, apesar de um número significativo de acidentes fatais na indústria de petróleo e gás (Hurtado, 2016) e uma expressiva presença no setor (Figueiredo, 2016) possua uma produção científica pouco relevante, tendo apenas $5 \%$ das publicações. A maioria são estudos da própria empresa de petróleo e gás em que houve a ocorrência de fatalidades, demonstrando a necessidade de mais estudos nacionais sobre o tema e da inclusão de novos interlocutores para refletir sobre o tema, tais como pesquisadores vinculados a universidades e centros de pesquisas.

Assim, pode-se afirmar, a partir da categorização e análise realizadas, que as publicações cientificas estão concentradas em um único periódico estadunidense. $O$ fato da revista ser sustentada por uma sociedade de profissionais da indústria do petróleo, de língua inglesa, além deter acesso restrito, pode constituir em fator limitante na disseminação do conhecimento a respeito do tema e também favorecer uma análise a partir do ponto de vista gerencial e/ou empresarial. De outro modo, observa-se uma escassez de pesquisas desenvolvidas pela área de Saúde Coletiva e por outros campos disciplinares que poderiam trazer olhares distintos e contribuir para ampliar e 
qualificar o debate sobre a ocorrência de acidentes graves na indústria de petróleo e gás.

\subsection{0 perfil dos acidentes fatais} que agravam a complexidade e aumentam a letalidade:

em uma plataforma podem se fazer presentes inúmeros fatores de riscos, tais como carga de trabalho excessiva, desenho inadequado de postos de trabalho, ruídos, vibrações, condições extremas de temperatura (frio e calor), ventilação inadequada, ar contaminado, gases, ácidos e vapores tóxicos e inflamáveis, além de outros produtos químicos com efeitos potencialmente deletérios para a saúde dos trabalhadores de plataformas. (...) Há, ainda, a possibilidade de ocorrência de: explosões, causadas por vazamentos de gases ou pressurização, além dos limites de ruptura de estruturas que os sustentam; incêndios; blow-out - vazamento súbito e incontido de petróleo e gás que pode ocorrer durante a perfuração de poços, podendo ter como consequência uma explosão devastadora, caso uma faísca entre em contato com o material que vazou; vazamento de gases tóxicos (benzeno, tolueno, xileno, ácido sulfídrico, amônia, monóxido de carbono etc.), colapso estrutural; choque elétrico; contato com superfícies frias ou quente demais; lesões de esmagamento causadas pela operação de máquinas ou pela queda de materiais; escorregões em superfícies pouco aderentes; afogamento por queda no mar (Figueiredo, 2016).

Uma hipótese para um maior número de fatalidades nas plataformas é que estas são instalações em alto mar, portanto com dificuldades de acesso, e abrigam sistemas tecnológicos complexos em um espaço compacto, aumentando a possibilidade de ocorrência de acidentes graves e com vítimas. Devemos salientar que os acidentes com helicóptero não entraram no cômputo dos acidentes em plataforma pelos autores dos estudos, embora tenham sido identificados 3 eventos com 58 óbitos. Em nosso entendimento, tais eventos (os acidentes de helicóptero) também estão diretamente 
relacionados a este tipo de instalação, pois é o meio de transporte para deslocamento entre a terra e o mar dos trabalhadores offshore.

Nas plataformas constata-se que as ocorrências tanto podem estar relacionadas a explosões e incêndios, como também à estabilidade da estrutura, tal como presença de rachaduras, além das colisões e naufrágio.

Em navios foram apenas duas ocorrências, uma delas com um número alto de mortes (193), sendo que as causas mais diretas dos acidentes foram naufrágio e colisão.

31 Identifica-se que nas refinarias, fábricas de fertilizantes e em petroquímicas são as explosões a principal causa dos acidentes fatais. Cabe sinalizar que nestas instalações os trabalhadores estão expostos a compostos químicos, contendo enxofre, nitrogênio, hidrocarbonetos aromáticos, monóxido de carbono, entre outros, e também a produtos gerados nestas unidades-petróleo, gasolina, nafta, querosene, amônia, os quais podem sofrer processo de combustão. Tais fatores têm sido detectados nas avaliações de riscos das instalações, através de inúmeras técnicas para identificação de perigos (Almeida \& Ferreira, 2008; Cardella, 2011), entretanto o conhecimento destes riscos não tem sido suficiente para eliminar as ocorrências de fatalidades nestas instalações. Estes tipos de eventos podem indicar que as situações degradadas das instalações e a ausência de barreiras de proteção eficazes, seja pela ausência de implantação ou por falha em sua atuação nas barreiras potencializam o risco das ocorrências.

32 Figueiredo, Alvarez \& Adams (2018) apontam em suas análises sobre os acidentes na indústria de petróleo e gás a existência de uma substancial e perigosa defasagem entre os avanços obtidos na gestão da inovação tecnológica e no gerenciamento de riscos. Assim, identifica-se que os avanços tecnológicos desta indústria não foram acompanhados da adoção de um conjunto de medidas (programas) capazes de lidar de modo eficaz, no decorrer do tempo, com a dinâmica dos riscos engendrados pelo funcionamento dos sistemas sociotécnicos complexos a ela associados. Aqui, é oportuno lembrar que, dadas as características de tais sistemas, múltiplas e inesperadas interações de falhas podem assumir uma configuração catastrófica (Perrow, 1999).

Em verdade, há situações em que o gerenciamento de riscos se mostra assaz retrógrado, em que a defasagem apontada no parágrafo anterior assume dimensões mais expressivas. Tal quadro se verifica, por exemplo, no caso de uma grande multinacional estudada por Figueiredo e Alvarez (2011). Uma empresa que atua como prestadora de serviços em regime de subcontratação para as grandes operadoras dessa indústria, e que é líder em seu ramo de atuação, inclusive no que tange aos aportes efetuados em P\&D. Malgrado sua condição, os trabalhadores não raramente são submetidos a arranjos completamente insólitos, expostos a situações nas quais devem se manter em estado de vigília por períodos bastante longos, por 24, 48, e até mesmo 72 horas ou mais! Isto porque a empresa não adota o esquema habitual de revezamento empregado em indústrias de processo contínuo, com duas equipes se alternando ao longo das 24 horas, cada qual cumprindo metade desta jornada (12 horas). Para subsidiar seus funcionários da maneira que julga mais adequada para lidar com tais circunstâncias, a empresa ministra conteúdos relacionados às 'técnicas de administração do sono'. Em tese, alguns dos recursos que os petroleiros devem lançar mão para enfrentar os limites que a necessidade de sono lhes impõe.

34 Constata-se, assim, a exposição de seus trabalhadores a condições que extrapolam os limites da jornada de $12 \mathrm{~h}$, com a empresa priorizando de modo flagrante o esforço de adaptação dos humanos a injunções inteiramente adversas, em frontal antagonismo aos 
preceitos elementares das chamadas abordagens clínicas do trabalho, e em total descompasso com a tecnologia de vanguarda empregada em seus processos. Não nos surpreende, portanto, que o maior índice de acidentes na empresa está relacionado à condução de veículos, às colisões que ocorrem após o cumprimento deste tipo de jornada (Figueiredo \& Alvarez, 2011). Note-se ainda que a tecnologia de vanguarda (de ponta) não impediu esta corporação de reproduzir os esquemas de precarização do trabalho tão recorrentes nas relações de subcontratação de serviços, em seus diversos níveis e modalidades.

O Quadro 1 apresenta um panorama das ocorrências identificadas, a data da ocorrência, o tipo de evento, o local ocorrido e o número de fatalidades.

Quadro 1- Descrição dos acidentes de trabalho na indústria de petróleo e gás de acordo com ano, tipo de planta, tipo de evento, local de ocorrência e número de óbitos

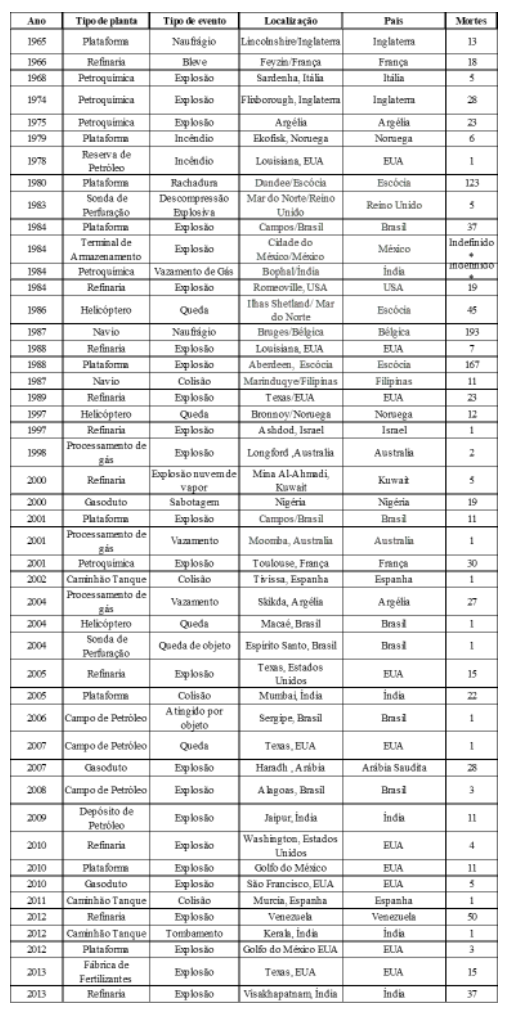

Smith (2002) aponta a relevância do tema dos acidentes de trabalho fatais para as próprias empresas de petróleo e gás do mundo e que, desde o ano de 1985, a Associação Internacional de Produtores de Petróleo e Gás (OGP) tem reportado publicamente informações sobre o desempenho de segurança e ocorrência de fatalidades nesta indústria.

37 A análise qualitativa dos estudos evidenciou três questões principais: a primeira referese à predominância dos acidentes de percurso ou típicos com veículos automotores. Em seguida, observa-se a preponderância das fatalidades envolvendo força de trabalho terceirizada e, em terceiro, as dificuldades em gerar aprendizado, a partir dos acidentes ocorridos, de modo a evitar novas ocorrências nesta indústria.

Em relação à alta taxa de acidentes com veículos automotores, Eckle e Burgherr (2013) realizaram estimativa de riscos das diferentes atividades da cadeia produtiva de óleo a partir da análise bayesiana de 1.200 acidentes fatais de países membros e não membros 
da OCDE ocorridos entre 1980 e 2008, de modo a identificar os principais riscos desta cadeia produtiva. Setenta por cento dos acidentes estavam relacionados a transporte e deslocamento e cerca de $60 \%$ dos acidentes ocorreram na Nigéria.

Igbuku (2007) apresenta resultado similar ao estudar a petrolífera Shell, onde os acidentes rodoviários são também a principal causa de mortes de seus trabalhadores, sejam próprios ou terceirizados. Este mesmo estudo aponta que o transporte rodoviário é compreendido como um modal de alto risco e gera alto custo socioeconômico.

Ainda de acordo com o estudo de Eckle e Burgherr (2013), na atividade de Exploração e Produção, a frequência de acidentes fatais é quase o dobro nos países não membros da OCDE quando confrontados aos países membros da OCDE. As ocorrências de taxas de acidentes no transporte terrestre também são bem mais expressivas nos países não membros da OCDE.

41 Corroboram esses resultados o estudo realizado por Covil e Smith (2002), ao realizar análise, a partir dos resultados do relatório de desempenho de segurança, denominado Health and Safety Performance of the Global E\&P Industry 2000, que identifica que as ocorrências com veículos automotores continuam sendo uma das principais causas de acidentes na área de Exploração e Produção. Este relatório assinala, por exemplo, que das 148 mortes reportadas à instituição, pelo menos 41 estavam relacionadas a acidentes de transporte, em especial com trabalhadores terceirizados, demonstrando a relevância dos resultados apresentados e que se assemelham aos reportados por Eckle e Burgherr (2013).

42 Ambituuni e colaboradores (2015a) analisaram acidentes relativos ao transporte rodoviário de produtos petrolíferos por caminhões-tanques na Nigéria. Este estudo identificou 2.318 acidentes envolvendo caminhões tanques no período de 2007 a 2012, que resultaram em 972 acidentes com morte de trabalhadores, a alta taxa de letalidade relacionada a acidentes de transporte nessa indústria.

Note-se ainda que Hill e colaboradores (2009) ao estudarem as taxas de fatalidade na indústria de extração de petróleo e gás norte-americana identificaram uma taxa de 30,5 mortes por 100.000 trabalhadores no período de 2003 a 2006, sendo 8 vezes maior que a taxa em todas as demais indústrias americanas. Nesse triênio houve o registro de 526 mortes de trabalhadores nesse setor, sendo que $29 \%$ destas mortes foram em decorrência de acidentes com transportes terrestres e $21 \%$ por impacto com objeto em movimento.

Covil e Smith (2002) analisando os relatórios de desempenho de Segurança na Indústria de Petróleo mundial divulgados pela OGP identificaram que o maior número de fatalidades ocorre com trabalhadores com vínculos mais precários (terceirizados, subcontratados, empreiteiros, entre outros). Os mesmos apontam que isto se dá porque são esses em geral os executantes das atividades operacionais de maior risco, além de possuírem menor qualificação e treinamento.

45 Figueiredo, Alvarez, Athayde, Suarez e Pereira (2007) também verificaram no setor offshore da Bacia de Campos no estado do Rio de Janeiro, Brasil, que cerca de $75 \%$ de sua força de trabalho é terceirizada e que a ocorrência de acidentes fatais atingindo estes trabalhadores é bem maior do que os próprios. Os autores referem-se à coexistência em um mesmo espaço de trabalho de duas categorias de trabalhadores, prevalecendo fortemente um quadro em que os trabalhadores da empresa (próprios) possuem maior estabilidade empregatícia, melhores salários e benefícios e uma formação mais 
consistente, enquanto os terceirizados, além da fragilidade de vínculo, são submetidos a jornadas de trabalho mais extenuantes, com menores períodos de repouso e, algumas vezes, treinamento inferior. Estas distinções trazem sérias consequências em relação às condições de trabalho e também à coesão e cooperação no trabalho, tão importantes para a proteção, saúde e segurança dos trabalhadores.

Dessa forma, ao intensificar em demasia a terceirização, sobretudo nas atividades de manutenção (...) a empresa abre um flanco para a desarticulação de um segmento importante de trabalhadores, alguns já detentores do saber (...). A despeito da capacidade de sustentação da confiabilidade pelos coletivos, entendemos que o risco introduzido ao se colocar em xeque sua coesão se configuraria como mais um elemento a respaldar a necessidade de um processo de reavaliação da forma com que a terceirização foi implantada (....) (Figueiredo, 2016)

Nessa linha, caberia acrescentar ainda que o fim do século passado foi marcado por uma expressiva reestruturação organizacional de grandes empresas petrolíferas no mundo, nos marcos de um novo modelo de organização com destaque para as unidades de negócio (UN's), em que se buscava atingir indicadores mais elevados de expansão, internacionalização, produtividade e rentabilidade. Nesse novo arranjo, o controle se dá de modo mais incisivo sobre o cumprimento de metas e resultados, possibilitando a essas subáreas menor constrangimento quanto ao gerenciamento de seus projetos e facilitando a diluição de responsabilidades. Esse modelo, calcado em tais UN's, já foi levado a cabo por diversas multinacionais do setor e é destacado por Le Coze (2016) em sua análise sobre o desastre envolvendo a plataforma Deepwater Horizon (no Golfo do México - EUA). Ele ressalta que com a criação das UN's nesta empresa, o coração do negócio da BP passou nitidamente da engenharia à gestão financeira e comercial de tais unidades, nas quais a terceirização foi amplamente adotada.

Sobre as lições aprendidas com os eventos, Denkl e colaboradores (2010) discutem que os acidentes se repetem ou sucedem eventos bastante semelhantes aos já ocorridos na indústria, o que sugere que o setor não tem sido capaz de aprender com as ocorrências pretéritas e, dessa forma, evitar que novas mortes de trabalhadores aconteçam. Assim, questiona-se o próprio processo de aprendizado gerado a partir de um evento passado. De acordo com Goyal (2010), sem a análise de falhas sistêmicas, de processos ou de projetos, não é possível propor medidas eficazes de longo prazo.

49 Leistad e Bradley (2009) acrescentam que se faz imperativo mudar o foco nas questões de acidentes nas instalações petrolíferas, pois segundo os autores não há de fato uma discussão ampla e direcionada para as situações que podem gerar acidentes ampliados e com número elevado de mortes, e são estes os responsáveis por cerca de $73 \%$ das mortes. Em geral, identifica-se uma intensificação de ações e até um esforço maior das empresas em modificar atitudes que poderiam levar a lesões pessoais e ao absenteísmo. Para estes autores deveria existir uma ação diferenciada, relativa às questões de Segurança de Processo, e que envolvem um risco maior para a totalidade dos trabalhadores da instalação, e ações mais simples, debaixo custo, e até mais eficazes, para os acidentes de menor potencial, mas de alta frequência. $O$ primeiro ocorre poucas vezes, mas quando ocorre impõe maiores riscos, maior número de óbitos e pode causar danos até à própria sobrevivência da empresa no mercado.

50 Egbeocha e colaboradores (2015) sublinham que a mudança etária da força de trabalho, isto é, a saída dos mais experientes e a entrada de jovens profissionais sem um planejamento adequado, pode comprometer o processo de transição e a passagem de 
conhecimento acarretando grandes riscos para a Segurança dos Processos das instalações, levando, por conseguinte, ao aumento dos acidentes de trabalho.

51 Cabral e colaboradores (2010) também identificaram como ponto crítico para a própria expansão das empresas petrolíferas a falta de profissionais qualificados e experientes, o que levou a maior empresa brasileira de energia a adotar em 2007 uma estratégia de contratação de trabalhadores novatos e a responsabilizar-se pelo treinamento destes profissionais, de modo que fossem capazes de realizar determinadas atividades críticas.

De um lado, verifica-se uma força de trabalho em fase de envelhecimento e aposentadoria e, por outro, a escassez de trabalhadores experientes e qualificados para a reposição. Assim, pensar em segurança e redução dos acidentes implica também em reconhecer que as lições aprendidas são o resultado do acúmulo de experiência, regras informais, saber tácito e que é necessário lançar mão de mecanismos para realizar este processo de aprendizado e tornar possível o acesso a este patrimônio longamente acumulado por aqueles que não vivenciaram tais ocorrências e que lidam com as vicissitudes do trabalho no dia a dia.

53 Uma observação de caráter geral é que as publicações sobre o tema são eminentemente desenvolvidas pelas empresas de petróleo e gás, evidenciando o ponto de vista do empregador e de seus gestores sobre as ocorrências e, desta forma, restringindo muitas vezes as análises às causas básicas e/ou imediatas, carecendo de análises ampliadas e multidisciplinares sobre a temática. Além disso, as informações sobre os agravos em decorrência de acidentes de trabalho não têm sido publicitadas e discutidas no âmbito dos serviços de atenção à saúde do trabalhador, o que compromete ações de vigilância em saúde.

\section{Considerações finais}

o quadro que se delineou no mundo com o expressivo desenvolvimento da indústria de óleo e gás, quando defrontado com os indicadores de acidentes das últimas décadas parece confirmar a existência de uma substancial e perigosa defasagem entre a gestão da inovação tecnológica e a gestão do risco.

A revisão bibliográfica sobre o tema acidente de trabalho fatal nessa indústria traçou o perfil das publicações nacionais e internacionais e uma análise crítica dos principais tipos e características dos acidentes que resultaram em mortes de trabalhadores ocorridos no setor. Ao trazer à luz uma análise das publicações identificadas e as características dos eventos descritos nos estudos publicados, espera-se contribuir para a reflexão e a disseminação do conhecimento produzido sobre os fatores e os mecanismos envolvidos na ocorrência desses agravos que possam contribuir para aumentar o conhecimento e reflexão sobre o tema. desenvolvimento de políticas e programas de saúde e segurança no trabalho e de transformações no setor.

Deve-se enfatizar ainda que diante da inversão da perspectiva ergonômica presente no caso da multinacional abordado anteriormente, somos obrigados a insistir na necessidade de uma profunda revisão de aspectos da gestão do trabalho, e do gerenciamento de riscos associado a esta, sob pena de introduzir-se mais um componente de risco dentre os inúmeros já presentes neste tipo de processo produtivo. 


\section{BIBLIOGRAFIA}

Almeida, E. P., \& Ferreira, M. L. R. (2008). Técnicas de análise de risco aplicadas à planejamento e programação de projetos da construção civil. Comunicação apresentada no IV Congresso Nacional de Excelência em Gestão, Niterói, Brasil, julho.

Ambituuni, A., Amezaga, J. M., \& Werner, D. (2015a). Risk assessment of petroleum product transportation by road: A framework for regulatory improvement. Safety Science, 79, 324-335.

Ambituuni, A., Amezaga, J. M., \& Werner, D. (2015b). Risk management framework for safe transportation of petroleum products in Nigeria: Learning from past accidents and good practices. Risk Management, 17, 4, 329-351.

Attwood, D., Khan, F., \& Veitch, B. (2006). Occupational accident models-Where have we been and where are we going? Journal of Loss Prevention in the Process Industries, 19, 6, 664-682.

Bariha, N., Mishra, I. M., \& Srivastava, V. C. (2016). Fire and explosion hazard analysis during surface transport of liquefied petroleum gas (LPG): A case study of LPG truck tanker accident in Kannur, Kerala. India Journal of Loss Prevention in the Process Industries, 40, 449-460.

Bhalla, J. (2010). Diesel engine runaway safety risk in hazardous environments (pp.464-478). In SPE Middle East Health, Safety, Security and Environment Conference and Exhibition. 464 - 478. Manama. 2018, de https://www.scopus.com/inward/record.uri?eid=2-s2.0-79952688642\&partnerID= 40\&md5=f5ccac1378229c42e2c6737d0878cfee.

Burgherr, P., Eckle, P., \& Hirschberg, S. (2012). Comparative assessment of severe accident risks in the coal, oil and natural gas chains. Reliability Engineering and System Safety, 105, 97-103.

Cabral, J. M., Pinheiro, F. M., Marrozzi, W. F., \& Marchi, L. C. (2010). Red alert program in drilling rigs: A strong decision to show leadership and to involve work force towards zero serious or fatal incidents. In SPE International Conference on Health, Safety and Environment in Oil and Gas Exploration and Production, 1648-1655, Brasil. 2018, de https://www.scopus.com/inward/record.uri?eid=2s2.0-77954223502\&partnerID=40\&md5=953d2b320f148eec $4164 a 19 \mathrm{e} 6 \mathrm{c} 202576$.

Cardella, B. (2011). Segurança no trabalho e prevenção de acidentes: uma abordagem holística. 1 ed. São Paulo: Atlas, CETES.

Catten, C., \& Johnson, C. (2014). The effect of management involvement on vehicle safety improvement. In SPE Production and Operations Symposium, 259-266, Estados Unidos. 2018, de https://www.scopus.com/inward/record.uri?eid=2-

s2.0-84931383649\&partnerID=40\&md5=eb73806510e234ab4010386ec6233d50.

Childress, G. W. (2014). The commentary climb: How to position intelligence over instinct. In SPE International Conference on Health, Safety, and Environment, 1336-1340, Estados Unidos. 2018, de https://www.scopus.com/inward/record.uri? eid=2s2.0-84905833496\&partnerID $=40 \& \mathrm{md} 5=07 \mathrm{f} 974 \mathrm{fbccf} 82266083 \mathrm{c} 6$ afd 5724ef5a.

Covil, M., \& Smith, D. (2002). Transportation Fatalities - An Industry Problem? In SPE International Conference on Health, Safety and Environment in Oil and Gas Exploration and Production, 1102-1105, Malásia. 2018, de https://www.scopus.com/inward/record.uri?eid=2s2.0-1642558312\&partnerID=40\&md5=b5df6594c8a 2294975191c5239ad49e7

Curlee, C. K., Broulliard, S. J., Marshall, M. L., Knode, T. L., \& Smith, S. L. (2005). Upstream onshore oil and gas fatalities: A review of OSHA's database and strategic direction for reducing fatal incidents. In SPE/EPA/DOE Exploration and Production Environmental Conference, 339-343, Estados 
Unidos. 2018, de https://www.scopus.com/inward/record.uri?eid=2-s2.0-22344448157\&partner ID $=40 \& m d 5=26 \mathrm{c} 1 \mathrm{e} 70776 \mathrm{f} 21 \mathrm{efeff} 010 \mathrm{e} 45 \mathrm{ea} 135 \mathrm{e} 31$.

Denkl, M., Anderson, M., \& Marley, B. (2010). Avoiding future fatal incidents through applying lessons from past knowledge. In SPE International Conference on Health, Safety and Environment in Oil and Gas Exploration and Production, 1070-1075, Brasil. 2018, de https://www.scopus.com/inward/ record.uri?eid=2-s2.0-77954231218\&partnerID=40\&md5=f06b254ff6d79fe87437c6f7d6f11e1e.

Ebrahimian, A., \& Stefanescu, E. (2011). HSE compliance in Romania. In SPE European Health, Safety and Environmental Conference in Oil and Gas Exploration and Production, 477-484, Aústria. 2018, de https://www.scopus.com/ inward/record.uri?eid=2s2.0-79959937844\&partnerID=40\&md5=465d50a315a 153ff7e4d732f257f78bb.

Eckle, P., \& Burgherr, P. (2013). Bayesian Data Analysis of Severe Fatal Accident Risk in the Oil Chain. Risk Analysis, 33, 1, 146-160.

Egbeocha, J. O., Reginald-Ugwuadu, O. G., Oluchi, E., Ebisike, R. C., \& Obanya, P. (2015).

Entrenching process safety culture in the face of shifting demography-A key to sustaining goal zero performance in well operations. In SPE Nigeria Annual International Conference and Exhibition, Nigéria. 2018, de https://www.scopus. com/inward/record.uri?eid=2-

s2.0-84958567923\&partnerID=40\&md5=c9d4fbd22 cef1e1630bf7c6590ae4f2a.

Espinosa, B. N., Azevedo, R. S., Turazzi, E., Glitz, A. G., Veloso, L. E. G., De Lacerda Filho, M. C., \& Bonaldo, N. C. (2010). HSE management tools for contractors, In SPE International Conference on Health, Safety and Environment in Oil and Gas Exploration and Production, 2102-2116, Brasil. 2018, de https://www.scopus. com/inward/record.uri?eid=2-

s2.0-77954294838\&partnerID=40\&md5=faabb1ea 02667cce8244a50ab45cd4f0.

Ezenwa, A. (2001). O. A study of fatal injuries in Nigerian factories.Occupational Medicine, 51, 8, 485489.

Ferreira, A. B. H. (2002). Aurelio o mini dicionário da língua portuguesa. (4. ed. ver. e ampl. do mini dicionário) Aurelio. Rio de Janeiro: Nova Fronteira.

Figueiredo, M. (2016). A face oculta do ouro negro: trabalho, saúde e segurança na indústria petrolífera offshore da Bacia de Campos. Niterói. EDUFF.

Figueiredo, M., \& Alvarez, D. (2011). Gestão do trabalho na perfuração de poços de petróleo: usos de si e 'a vida por toda a vida'. Trabalho, Educação e Saúde, 9(Suppl. 1), 299-326. https://dx.doi.org/ $10.1590 /$ S1981-77462011000400015

Figueiredo, M., Alvarez, D., \& Adams, R. (2018). O acidente da plataforma de petróleo P-36 revisitado 15 anos depois: da gestão de situações incidentais e acidentais aos fatores organizacionais. Cadernos de Saúde Pública, 34(4), e00034617. Epub March 29, 2018.https:// dx.doi.org/10.1590/0102-311x00034617

Figueiredo, M., Alvarez, D., Athayde, M., Suarez. J. D., \& Pereira R. (2007). Reestruturação produtiva, terceirização e relações de trabalho na indústria petrolífera offshore da Bacia de Campos (RJ). Gestão e Produção, 14, 55-68.

Fullerton, L., Olson, L., Crandall, C., Sklar, D., \& Zumwalt, R. (1995). Occupational Injury Mortality in New Mexico. Annals of Emergency Medicine, 26, 4, 447-454.

Goyal, R. K. (2010). Lessons learned from process incidents - Viewing historical data through the lens of 'tenets of operational excellence'. In Middle East Health, Safety, Security, and Environment Conference and Exhibition, 507-518, Barém. 2018, de https://www.scopus.com/inward/record.uri? eid=2-s2.0-79952685384\& partnerID=40\&md5=8815bf9b12fc31dba2d67dec1af31366>. 
Hill, R. D., Somervell, P. D., \& Conway, G. A. (2009). Injury risk among oil and gas extraction workers by company type and size. In SPE Americas E\&P Environmental and Safety Conference, 447451, Estados Unidos. 2018, de https://www.scopus.com/inward/record.uri?eid=2s2.0-70349947007\&partnerID $=40 \& m d 5=705002 f 03 c 6 e 79 \mathrm{e} 2 \mathrm{bca} 4 \mathrm{~b} 211 \mathrm{ebe} 074 \mathrm{e} 2>$.

Hought, J. (2010). Amnesia blights process safety. Process Engineering (London), 91, 4, 8-10.

Hurtado, S. L. B. (2016). Análise de Acidente de Trabalho em uma refinaria de petróleo: desencontros entre a segurança comportamental e a organizacional. Dissertação de Mestrado. Universidade de São Paulo, São Paulo.

Igbuku, A. O. (2007). Integration of hearts and minds concept and road safety enforcement for accident reduction. In SPE Asia Pacific Health, Safety, and Security Environment Conference and Exhibition, 175-180, Tailândia, 2018, de https://www.scopus.com/inward/record.uri?eid=2s2.0-58149470597\&partnerID= 40\&md5=5acb7470d06c7b51cba67412dbd8fb0c.

Instituto Brasileiro de Petróleo, Gás e Biocombustível. (2016). Maiores produtores mundiais de petróleo em 2016. 2018, dehttps://www.ibp.org.br/observatorio-do-setor/maiores-produtores-depetroleo-e-lgn-em-2016/

Ismail, Z., Ramli, A. H., \& Somarin, S. D. (2012). Lessons learned from accidents in downstream petroleum product processing and handling. Oil Gas European Magazine, 38, 3, 157-162.

Jutten, J. J., Ndubisi, J. J., Regnault, P., \& Abifarin, O. (2002). Working toward Zero Automotive Accidents in the Niger Delta. In SPE International Conference on Health, Safety and Environment in Oil and Gas Exploration and Production, 1113-1118, Malásia. 2018, de https://www.scopus.com/inward/ record.uri?eid=2-s2.0-1642558311\&partnerID=40\&md5=dcd0ca3dafedcd4836308b982cd9cdb1.

Khan, F. I., \& Abbasi, S. A. (1999). The world's worst industrial accident of the 1990s: What happened and what might have been - A quantitative study. Process Safety Progress, 18, 3, 135-145.

Le Coze, J.C. (2016). Le nouveau visage des risques socio-technologiques. Toulouse: Octarès.

Leistad, G. H., \& Bradley, A. R. (2009). Is the focus too low on issues that have a potential to lead to a major incident? In Offshore Europe, 467-472, Escócia. 2018, de https://www.scopus.com/inward/

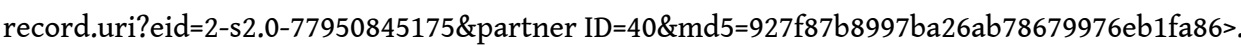

Lightfoot, R. M., \& Susler, O. (2006). Occupational health and safety profiling. In SPE International Conference on Health, Safety and Environment in Oil and Gas Exploration and Production, vol. 8, 10071012, Emirados Árabes. 2018, de https://www.scopus.com/inward/record.uri?eid=2s2.0-33745339800\&partnerID $=40 \& \mathrm{md} 5=3 \mathrm{aa} 3 \mathrm{e} 40 \mathrm{c} 265 \mathrm{f} 79 \mathrm{da} 443451 \mathrm{~b} 438 \mathrm{dcd} 9 \mathrm{f} 8$.

Lindøe, P. H., Engen, O. A., \& Olsen, O. E. (2011). Responses to accidents in different industrial sectors. Safety Science, 49, 1, 90-97.

Martinez, J. M. B. (2013). Liquefied natural gas road tanker explosion. In IGT International Liquefied Natural Gas, vol. 17, 26-38, Estaos Unidos. 2018, de https://www.scopus.com/inward/record.uri? eid=2-s2.0-84880621669\&partnerID= 40\&md5=d10b18eba5c3bf45094607e05195da7a.

Ministério da Fazenda. (2015). Anuário Estatístico da Previdência Social. 2018, de http:// www.previdencia.gov.br/wp-content/uploads/2015/08/AEPS-2015-FINAL.pdf

Odunmbaku, S., \& Obarewon, F. (2007). Reducing road traffic fatalities through driver education: A major oil-producing company's approach. In SPE E and P Environmental and Safety Conference 2007: Delivering Superior Environmental and Safety Performanc, 98-101, Estados Unidos. 2018, de https:// www.scopus. com/inward/record.uri?eid=2-s2.0-34249040854\&partnerID=40\&md5=e9e4e388 cbaa0b9c8720bd25265df611. 
Organização Internacional do Trabalho (2013). A prevenção das enfermidades profissionais. Genebra. 2018, de http://www.ilo.org/wcmsp5/groups/public/---ed_protect/---protrav/---safework/ documents/publication/wcms_209555.pdf

Organização Internacional do Trabalho. (1981). Conferência Internacional do Trabalho. Convenção 155. Genebra.

Perrow, C. (1999). Normal Accidents: Living with High-Risk Technologies. New Jersey: Princeton University Press.

Piccinini, N., \& Demichela, M. (2012). Five dead and five injured in a dimethyl terephthalate plant accident: Serious errors in the plant design coupled with incorrect maintenance management. Industrial and Engineering Chemistry Research, 51, 22, 7619-7627.

Powell, T. (1996).obtaining real improvement in health \& safety performance requires force. In International Conference on Health, Safety and Environment in Oil and Gas Exploration and Production, 55-65, Estados Unidos. 2018 de https://www.scopus.com/inward/record.uri?eid=2s2.0-0029722551\&partnerID $=40 \& \mathrm{md} 5=84 \mathrm{a} 84 \mathrm{db} 265 \mathrm{dfa} 18 \mathrm{ec} 59 \mathrm{f} 7977 \mathrm{c} 3065 \mathrm{c} 6 \mathrm{c}>$.

Riezel, Y. (2002). Explosion and fire in a gas-oil fixed roof storage tank: Case study and lessons learned. Process Safety Progress, 21, 1, 67-73.

Santana V. S., Araújo Filho J. B., Silva M., Oliveira P. R. A., Branco A., \& Nobre L. (2007).

Mortalidade, anos potenciais de vida perdidos e incidência de acidentes de trabalho na Bahia, Brasil. CadSaúdePública, 23, 11, 2643-52.

Schmidt, V. (2013). Safety becomes cool. Offshore Engineer, 38, 2, 27-29.

Sharma, R. K., Gurjar, B. R., Singhal, A. V., \& Agrawal, R. (2015). Automation of emergency response for petroleum oil storage terminals. Safety Science, 72, 262-273.

Sherman, C. (2009). Offshore diving safety - Challenges and concerns. In Offshore Europe Oil and Gas Conference and Exhibition, 335-340, Escócia. 2018, de https://www.scopus.com/inward/record.uri? eid=2-s2.0-77950847404\&partner ID=40\&md5=f0aa504094f8d60958a2376d72432d8a .

Skelton, D. B. (2014) Examining the use of blast resistant modules (BRMs) Within API 753 zones 1 and 2. In Center for Chemical Process Safety International Conference, vol. 29, 583-593, Estado Unidos. 2018, de https://www.scopus.com/inward/record.uri?eid=2-s2.0-84909960120\&partnerID= 40\&md5=2ab7011903b45dd0e34b466932672e 73 .

Smith, D. (2002). Health and Safety Performance of the Global E\&P Industry 2000. In Offshore Technology Conference, 1431-1440, Estados Unidos. 2018, de https://www.scopus.com/inward/ record.uri?eid=2-s2.0-1642562906\&partnerID $=40 \& \mathrm{md} 5=\mathrm{c} 0 \mathrm{f} 54 \mathrm{f} 38 \mathrm{a} 2963 \mathrm{~b} 1 \mathrm{a} 0 \mathrm{fc} 8 \mathrm{~d} 5 \mathrm{c} 2 \mathrm{c} 9 \mathrm{a} 51505$.

Stuart, C., \& Foo, S. (2010) Application of an intelligent system to ensure integrity throughout the entire well life cycle. In IADC/SPE Asia Pacific Drilling Technology Conference, 542-559, Vietname. 2018, de https://www.scopus.com/ inward/record.uri?eid=2s2.0-79953069755\&partnerID=40\&md5=a99f380d89 02ed74b6468b882df7b873.

Threadgold, I., Jeffrey, C., Sixma, E., \& Garcia, D. (2010). Working together - Saving lives. In SPE International Conference on Health, Safety and Environment in Oil and Gas Exploration and Production, 1364-1369, Brasil. 2018, de https://www.scopus.com/inward/record.uri?eid=2s2.0-77954225692\&partnerID=40\&md5=33903e3458a691dc8b79f4eedf1b039a .

Valner, J., \& Leighton, S. J. (2009). Validation of EGNOS helicopter approach procedures to North Sea Oil platforms. Journal of Navigation, 62, 2, 189-201. 
Wilson, J. E., \& Gallagher, J. (2005). Cleanup of a 32,000-barrel crude oil spill at the west hackberry dome storage site in Louisiana. In International Oil Spill Conference, 1083, Estados Unidos. 2018, de +https://www.scopus.com/inward/record.uri?eid=2s2.0-3646017081\&partnerID=40\&md5=42a294221157182047c 9f5423b604b95 .

\section{RESUMOS}

Este artigo tem como objetivo identificar e analisar criticamente o perfil dos acidentes de trabalho fatais na indústria de petróleo e gás a partir de literatura científica disponível eletronicamente sobre o tema. Foi realizada revisão bibliográfica do tipo narrativa utilizando a base de dados Scopus. A busca foi desenvolvida no mês de outubro de 2016, sem restrição temporal, excluindo doenças e comorbidades. Foram identificados 198 artigos e destes foram selecionados e analisados na sua íntegra 42 artigos. Os acidentes reportados ocorreram no período de 1965 a 2013. Há predominância dos estudos publicados nos EUA, uma alta concentração em um único periódico da área de Engenharia e escassez de publicações na área da Saúde. Observou-se nos últimos anos uma tendência de crescimento das taxas de acidentes de trabalho fatais em países como na Nigéria. A maioria dos acidentes ocorreu em plataformas, navios e refinarias, totalizando 1043 fatalidades de trabalhadores no período estudado, com pico de óbitos nos anos 1980.

Ese artículo tiene como objetivo identificar y analizar críticamente el perfil de los accidentes de trabajo fatales en la industria del petróleo y gas a partir de la literatura científica disponible electrónicamente sobre el tema. Fue realizada revisión bibliográfica del tipo narrativo utilizando la base de datos Scopus. La busca fue efectuada en el mes de octubre de 2016, sin restricciones temporales, excepto enfermedades y patologías asociadas. Fueron identificados 198 artículos y de estos fueron seleccionados y analizados en la íntegra 42 artículos. Los accidentes reportables ocurrieron en el período de 1965 a 2013. Hay predominancia de los estudios publicados en los Estados Unidos de América una alta concentración en un único periódico del área de ingeniería y escasez de publicaciones en el área de salud. Se observó en los últimos años una tendencia de crecimiento de los índices de accidentes de trabajo fatales en países como en Nigeria. La mayoría de los accidentes ocurrió en plataforma, navíos y refinerías, totalizando 1043 fatalidades de trabajadores en el periodo estudiado con pico de óbitos en los años 1980.

Cet article a pour objectif d'identifier et analyser le profil des accidents du travail mortels dans l'industrie du pétrole et du gaz à partir de la littérature scientifique disponible électroniquement sur le thème. On a effectué une revue narrative à partir de la base de données Scopus. La recherche a été développée en octobre 2016, sans restriction temporelle, en excluant les maladies et comorbidités. On a identifié 198 articles, dont 42 ont été sélectionnés et analysés intégralement. Les accidents rapportés ont eu lieu entre 1965 et 2013. Il y a prédominance d'études publiées aux États-Unis, une forte concentration dans une seule revue d'Ingénierie et peu de publications dans le domaine de la Santé. On a observé au cours des dernières années une tendance à la croissance des taux d'accidents du travail mortels dans des pays comme le Nigeria. La majorité des accidents a eu lieu sur des plateformes, dans des raffineries et des navires pétroliers, totalisant 1043 décès de salariés au cours de la période étudiée, avec un pic de décès dans les années 1980.

This article aims to identify and critically analyze the profile of fatal work accidents in the oil and gas industry from electronically available scientific literature on the subject. A narrative bibliographic survey was carried out using the Scopus database. The search was hold on October 2016, with no temporal restriction and excluding diseases and comorbidities. There were 198 
identified articles and 42 were selected and fully analyzed. The reported accidents had happened between the years of 1965 and 2013. There is a predominance of studies released in the USA; high concentration of publications in a single journal of the Engineering area; shortage of publications in the area of Health. In the last few years, it was notorious a rising fatality rate in countries such as Nigeria. Most of the occupation injuries occurred in platforms, ships and refineries, resulting in the death of approximately 1043 people, mostly in the 1980 s.

\section{ÍNDICE}

Keywords: oil and gas industry, work accidents, occupational mortality, literature review, occupational health

Palavras-chave: indústria de petróleo e gás, acidentes de trabalho, mortalidade ocupacional, revisão de literatura, saúde do trabalhador

Palabras claves: industria del petróleo y gas, accidentes de trabajo, mortalidad ocupacional, revisión de literatura, salud del trabajador

Mots-clés: industrie du pétrole et du gaz, accident de travail, mortalité au travail, revue de la littérature, santé du travailleur

\section{AUTORES}

\section{HILKA GUIDA}

Escola Nacional de Saúde Pública (ENSP)

Fundação Oswaldo Cruz (FIOCRUZ)

Rua Leopoldo Bulhões, 1480, sala 302, Manguinhos - Rio de Janeiro - RJ. Brasil, CEP: 21041-210

hilka.guida@yahoo.com.br

\section{ÉLIDA AZEVEDO HENNINGTON}

Centro de Estudos da Saúde do Trabalhador e Ecologia Humana (CESTEH)

Escola Nacional de Saúde Pública (ENSP)

Fundação Oswaldo Cruz (FIOCRUZ)

Rua Leopoldo Bulhões, 1480, sala 302, Manguinhos - Rio de Janeiro - RJ. Brasil, CEP: 21041-210

elidahennington@gmail.com

\section{MARCELO FIGUEIREDO}

Núcleo de Estudos em Inovação, Conhecimento e Trabalho (Neict)

Departamento de Engenharia de Produção - Escola de Engenharia

Universidade Federal Fluminense (UFF)

Av. Passo da Pátria, 156, Niterói, RJ - Brasil, CEP: 24210-240

marceloparada@uol.com.br 